\title{
Re-evaluating the relationships among filtering activity, unnecessary storage, and visual working memory capacity
}

\author{
Stephen M. Emrich • Michael A. Busseri
}

Published online: 18 February 2015

(C) Psychonomic Society, Inc. 2015

\begin{abstract}
The amount of task-irrelevant information encoded in visual working memory (VWM), referred to as unnecessary storage, has been proposed as a potential mechanism underlying individual differences in VWM capacity. In addition, a number of studies have provided evidence for additional activity that initiates the filtering process originating in the frontal cortex and basal ganglia, and is therefore a crucial step in the link between unnecessary storage and VWM capacity. Here, we re-examine data from two prominent studies that identified unnecessary storage activity as a predictor of VWM capacity by directly testing the implied path model linking filtering-related activity, unnecessary storage, and VWM capacity. Across both studies, we found that unnecessary storage was not a significant predictor of individual differences in VWM capacity once activity associated with filtering was accounted for; instead, activity associated with filtering better explained variation in VWM capacity. These findings suggest that unnecessary storage is not a limiting factor in VWM performance, whereas neural activity associated with filtering may play a more central role in determining VWM performance that goes beyond preventing unnecessary storage.
\end{abstract}

Keywords Working memory $\cdot$ Attention $\cdot$ ERP $\cdot$ Short-term memory $\cdot$ fMRI

Over the past few decades, there has been enormous interest in understanding the nature of visual working memory (VWM), which enables the on-line maintenance of a limited amount of visual information over short periods of time. In particular, a

S. M. Emrich $(\bowtie) \cdot$ M. A. Busseri

Department of Psychology, Brock University, 500 Glenridge Ave, St

Catharines, Ontario L2S 3A1, Canada

e-mail: semrich@brocku.ca number of studies have focused on understanding the origins of individual differences in VWM capacity (Luck \& Vogel, 2013). That is, VWM has a limited bandwidth, and as such, the number of items (capacity) and/or the fidelity of information stored in VWM appear to be severely limited, with significant individual differences in this ability.

Although the precise origins of individual differences in VWM capacity remains unclear, one mechanism that has been proposed to account for these differences is the ability to efficiently allocate limited capacity resources through the filtering of unnecessary (distractor) information, thereby minimizing the unnecessary storage of task-irrelevant information (Awh \& Vogel, 2008; Luck \& Vogel, 2013). Specifically, if VWM is characterized as having a limited number of storage "slots," then any item that is encoded into VWM will occupy one of these storage units. According to this model, the more control you have over which information gains access to VWM, the less likely you are to encode task-irrelevant information that will occupy these limited storage slots, thereby freeing up these resources for the task-relevant items. This efficient allocation of capacity-limited resources has been linked to VWM capacity, as shown by a positive correlation between measures of filtering efficiency and VWM capacity (Vogel, Mccollough, \& Machizawa, 2005). From this perspective, filtering efficiency can be indexed by the amount of unnecessary activity - that is, the amount of additional maintenance-related activity in the presence of distractors compared to conditions without distractors. Those individuals who demonstrate less unnecessary storage also tend to have higher VWM capacity, suggesting that the control over what information is encoded into VWM may be an important determinant of individual differences in VWM capacity.

This account emphasizing filtering ability as a primary predictor of individual differences in VWM capacity has been extremely influential (Luck \& Vogel, 2013). However, despite the emphasis that has been placed on the role of unnecessary 
storage, its exact contribution to individual differences remains somewhat unclear. Here, based on a re-analysis of two studies examining neural activity associated with filtering preparedness or initiation, as well as unnecessary storage (Liesefeld, Liesefeld, \& Zimmer, 2014; McNab \& Klingberg, 2008), we re-examine the evidence in support of unnecessary storage as a predictor of VWM capacity by testing the implied path model linking activity associated with filtering, unnecessary storage, and VWM capacity. Our findings reveal that unnecessary storage is a poor predictor of individual differences in VWM capacity when examined alongside activity driving the filtering process. Instead, activity associated with attentional control (i.e., the activity that initiates the filtering of irrelevant distractors) uniquely predicts VWM capacity independent of unnecessary storage.

\section{The bouncer in the brain}

The relationship between filtering ability and VWM capacity was first identified in a study by Vogel and colleagues (Vogel et al., 2005), who demonstrated that low-capacity individuals (as opposed to high-capacity individuals) were more affected by task-irrelevant distractors presented during encoding, and that these low-capacity subjects also exhibited greater contralateral delay activity (CDA) in the presence of distractors. From an information processing perspective, the objective for the observer should be to efficiently allocate limited VWM resources by preventing irrelevant information from being unnecessarily represented in VWM. Given that the CDA is an event-related potential (ERP) associated with VWM maintenance (McCollough, Machizawa, \& Vogel, 2007), these low-capacity subjects appear to have encoded more task-irrelevant distractors than did high-capacity subjects. That is, greater neural measures of VWM maintenance in the presence of task-irrelevant distractors (namely, unnecessary storage) is indicative of a "leaky filter" in these lowcapacity subjects.

Such negative correlations between unnecessary storage and VWM capacity has led to the suggestion that individual differences in the ability to effectively allocate capacitylimited resources through the filtering of irrelevant information may be a determining source of variation in overall VWM capacity. Awh and Vogel (2008) compared this ability to a bouncer at a nightclub. According to this view, individuals with higher (vs. lower) capacity have bouncers that are better able to keep unwanted patrons (i.e., task-irrelevant information) from entering the nightclub. By keeping unwanted patrons out, the nightclub is less likely to be overloaded and overworked by unwanted guests (i.e., irrelevant information). In other words, better performance could be attributed to a better bouncer, rather than to a larger nightclub per se.
Importantly, although these findings suggest that attentional selection mechanisms, rather than storage capacity, may determine VWM capacity (Luck \& Vogel, 2013), this notion has largely been inferred based on the association between unnecessary storage and VWM capacity. That is, the role of the "bouncer" (the attentional selection mechanism) has been observed primarily through its role in preventing irrelevant information from occupying capacity-limited resources. It is clear how this sort of filtering mechanism would lead to better VWM performance in the presence of distractors: By preventing the selection and encoding of task-irrelevant distractors, more resources can be allocated to the task relevant information. Less clear, however, is how this selection mechanism (namely the mechanism used to prevent unnecessary storage) could affect VWM capacity in the absence of distractors. That is, how does a mechanism that prevents the storage of irrelevant distractors determine VWM capacity when no distractors are present?

One possibility is that the activity associated with the selection and filtering of information, rather than unnecessary storage per se, is a more central factor determining VWM capacity. In other words, unnecessary storage may correlate with individual differences in VWM capacity not because the allocation of capacity-limited resources is what drives VWM performance, but rather because unnecessary storage reflects other processes that drive selection and encoding more generally (i.e., attentional selection mechanisms). One way to test this possibility is determine whether the misallocation of resources (as measured by unnecessary storage) still predicts VWM capacity once neural activity associated with the filtering process is accounted for. That is, if we can more directly measure the attentional selection mechanisms driving filtering, does this activity explain both unnecessary storage and individual differences in VWM capacity? Here, we address this question by re-analyzing data from two studies that examined associations among individual differences in filteringrelated activity, unnecessary storage, and VWM capacity.

\section{Study 1: Reanalysis of McNab and Klingberg (2008)}

In the first study, we re-examined the results of an influential fMRI study by McNab and Klingberg (2008) examining correlations between the neural measures of filtering set activity, the neural measure of unnecessary storage activity, and VWM capacity. For full methods including fMRI scanning parameters, see McNab and Klingberg (2008).

Summary of the original study

In the original study by McNab and Klingberg (2008), 25 participants performed a working memory task while undergoing fMRI. Participants were presented with a variable 
number of red and yellow circles to remember, presented for 1 s. Prior to the presentation of the memory sample, participants were presented a display (for between 3 and $5 \mathrm{~s}$ ) instructing them either to remember all of the items (no distraction) or only the red items (the "distraction" condition). Thus, participants were instructed to hold in memory either three red circles while ignoring two yellow circles (the "distraction" condition), or to remember three or five items, including in some cases the yellow circles. After a delay of 2-4 s, participants had to indicate whether a probe item was presented in the location of one of the target items.

fMRI BOLD signals were modeled using a canonical hemodynamic response, and examined for two types of activity. Filtering set activity was defined by contrasting activity observed during the instruction cue in the distraction compared to the no distraction trials. That is, separate regressors were used for each of the instruction conditions, for the sample presentation (with a covariate for the number of items presented during the sample), and regressors for both the maintenance and storage phases. Thus, this activity was thought to reflect the preparation to filter out distractors, and was observed in bilateral regions of the prefrontal cortex (middle frontal gyrus) and the left basal ganglia (with peaks in the putamen and global pallidus). Unnecessary storage activity was defined as the amount of activity in load-sensitive regions of parietal and occipital cortices in the presence of distractors compared to when no distractors were present. In this case, separate regressors were used for each of the instruction displays, and for each of the distraction and no-distraction conditions, as well as the response period. Specifically, the authors isolated the regions of parietal and occipital cortex by comparing delay-period activity in load 5 and load 3 trials in the no-distraction condition. Unnecessary storage activity was then calculated by comparing the load 3 no-distraction conditions to the load 3 distraction conditions (which included two yellow distractors). Thus, any increase in activity in these areas in the presence of the distractors was thought to reflect the number of task-irrelevant distractors stored in VWM compared to when no distractors were present.

Importantly, VWM capacity was measured using a variant of the no-distraction task outside of the scanner, and calculated using the Pashler-Cowan formula (Cowan, 2001) following the presentation of the stimuli.

Of interest here were the observed relationships between neural measures associated with filtering set activity and unnecessary storage, and the behavioral measure of VWM capacity. The authors observed that filtering set activity in the PFC and basal ganglia was positively correlated with VWM capacity, and filtering set activity in the globus pallidus region of the basal ganglia (but not activity in the PFC) was negatively correlated with unnecessary storage. Further, unnecessary storage was negatively correlated with VWM capacity. According to the study authors, the patterns of correlations were "consistent with the hypothesis that unnecessary storage accounts for the correlation between filtering set activity and working memory capacity" (McNab \& Klingberg, 2008, p. $105)$. This account thus positions unnecessary storage as the intervening mechanism between filtering set activity and VWM capacity (i.e., filtering set activity $\rightarrow$ unnecessary storage $\rightarrow$ VWM capacity).

This explanation has important conceptual and empirical implications for understanding individual differences in VWM capacity. Conceptually, the filtering mechanism determines the efficiency of VWM through preventing irrelevant information from occupying capacity-limited resources (i.e., unnecessary storage). Empirically, the model proposed by McNab and Klingberg (2008) implies that preparatory filtering activity has no unique or direct impact on VWM capacity independent of unnecessary storage. Rather, the effect of filtering set activity should be fully explained by unnecessary storage such that unnecessary storage would predict VWM independent of filtering set activity, but filtering would not predict VWM capacity independent of unnecessary storage. However, this assumption was not tested directly in the original study. For the present re-analysis, we evaluated the path model implied by the proposed mechanism, as well as a modified model in which both filtering set activity and unnecessary storage predicted VWM capacity.

\section{Reanalysis}

We first evaluated a path model using AMOS software in which filtering set activity in the globus pallidus region of the basal ganglia predicted unnecessary storage activity, and unnecessary storage activity predicted VWM capacity. Results are shown in Fig. 1A. Note that the standardized path coefficients shown in this figure are identical to the observed pairwise correlations between adjacent variables because each path in the model represents a regression of one criterion variable on one predictor. Critically, this model provided poor fit to the data $\left(\chi^{2}=5.54, d f=1, p=.02 ; \mathrm{CFI}=.68\right.$; RMSEA $=$ $.44, p$ for close fit $=.02$ ) owing to a large residual association between filtering set activity and VWM capacity not accounted for by the model as specified. Thus, a modified model was tested in which an additional direct path from filtering set activity to VWM capacity was included. Because this model was just-identified (i.e., $d f=0$ ), it necessarily provided a perfect model fit. More importantly, results from this model (see Fig. 1B) indicated that filtering set activity had a unique direct effect on VWM capacity $(p=.01)$, whereas the direct predictive effect of unnecessary storage was nonsignificant $(p=.31)$. Critically, this model explained nearly twice the variance in VWM capacity $\left(R^{2}=.35\right)$ than the original model $\left(R^{2}=.18\right)$.

These results indicate that unnecessary storage did not account for the association between filtering set activity and 

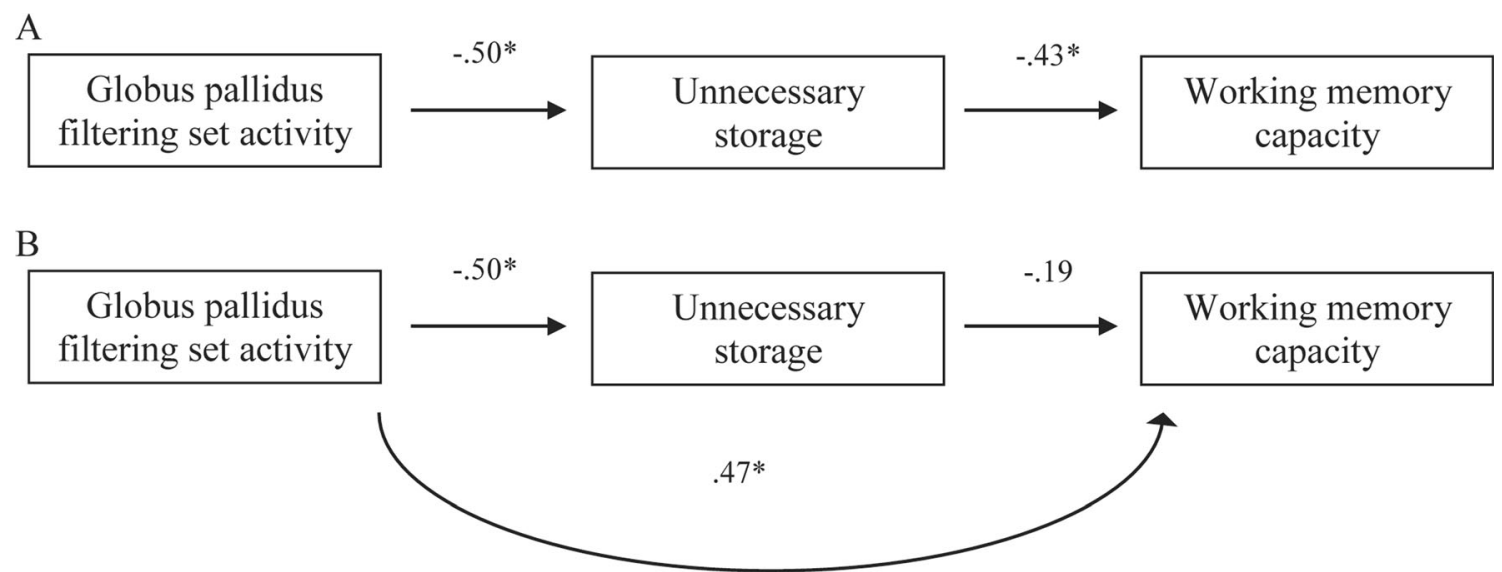

Fig. 1 Results from the re-analysis of the findings presented by McNab and Klingberg (2008). Panel A shows results from the causal model proposed by the study authors. Panel B shows results from the modified model. Standardized path coefficients are shown. ${ }^{*} \mathrm{p}<.05$

VWM capacity. Further, unnecessary storage activity was not a unique predictor of VWM capacity independent of filtering set activity. However, filtering set activity was a unique predictor independent of unnecessary storage. Thus, rather than revealing a neural mechanism whereby filtering activity is linked with VWM capacity via the amount of unnecessary storage, the revised findings suggest that the neural activity underlying filtering ability has a direct predictive effect on both unnecessary storage and VWM capacity. Moreover, contrary to the conclusions of McNab and Klingberg (2008), unnecessary storage had no unique predictive effect on VWM capacity.

\section{Study 2: Reanalysis of Liesefeld et al. (2014)}

A recent study by Liesefeld et al. (2014) provides additional insight into the puzzle concerning the successful operation of filtering mechanisms in VWM, through examining correlations among electrophysiologic measures of three filteringrelated processes (distractor detection, filtering initiation, and unnecessary storage) and VWM capacity. For a detailed description of methods, see Liesefeld et al. (2014).

\section{Summary of original study}

In the original study by Liesefeld et al., 40 participants performed a lateralized change-detection task while EEG was recorded. Each trial began with an arrow cue, lasting for 200 $\mathrm{ms}$, that cued one side of the display to be attended. Following the cue, colored squares and rectangles were presented for 100 $\mathrm{ms}$, following a 100 - to $200-\mathrm{ms}$ fixation. On some trials, only targets (e.g., squares) were presented, whereas on other trials both targets and distractors (e.g., rectangles) were presented. Participants were instructed to remember the colors of the squares (targets) while ignoring the rectangles (distractors). The number of targets and distractors varied (two to five targets only, or two targets with two or three distractors, and three targets with two distractors). Thus, by varying independently the number of targets and distractors, the authors were able to isolate independently the effects of (1) the total number of items, (2) the number of targets, and (3) the number of distractors. After a 900-ms delay, participants had to detect the presence or absence of a change in the target (square) items on the cued side of the display.

Similar to McNab and Klingberg (2008), the analysis by Liesefeld et al. focused on two main contrasts. The distractorpresence contrast compared trials with four or five targets only to those trials with distractors. Thus, this comparison could isolate the effect of distractors on electrophysiologic components while controlling for the total number of items. This analysis isolated two separate components. The earliest component was the distractor detection component, which was observed in posterior electrodes, and showed larger peak amplitude in the presence of distractors. This component occurred over contralateral (attended) channels about $228 \mathrm{~ms}$ after the onset of the sample display, and showed larger amplitudes for trials with distractors than those trials with only targets, suggesting a role in the initial detection of distractors in the display. Following the distractor detection component was a component that occurred over frontal electrode sites, and which was affected primarily by the presence or absence of distractors from the sample array. The authors hypothesized that this second component - a "prefrontal bias signal" reflected the initiation of filtering processes once the distractors were identified by the posterior distractor detection processes.

The second analysis by Liesefeld et al. focused on isolating the effects of unnecessary storage. This was achieved by isolating the parietal components that increased as a function of the number of objects, and was sustained throughout the delay. This analysis revealed a slow-wave component that occurred over posterior electrode sites and was affected by the number of targets as well as distractors, and is similar to the 
CDA. Slow-wave parietal activity was then compared between target-only trials and distractor-present trials with the same number of targets. Thus, this unnecessary storage activity is thought to reflect the additional number of taskirrelevant distractors stored in VWM.

Liesefeld et al. found that distractor detection latency was negatively correlated with prefrontal bias signal and VWM capacity, and positively correlated with unnecessary storage. Prefrontal bias signal strength was negatively correlated with unnecessary storage, and positively correlated with VWM capacity. Further, unnecessary storage was negatively correlated with VWM capacity. Thus, the authors proposed a three-stage model of efficient filtering, whereby faster detection of distractors (as indicated by posterior EEG components) leads to a stronger bias signal from the prefrontal cortex triggering the filtering process (observed as a frontal ERP that preceded the onset of unnecessary storage activity), which then enables greater exclusion of distractors from VWM and thus less unnecessary storage (as assessed by parietal delay activity similar to the CDA). Together, these three processes are thought to form a "causal chain of events that constitute filtering in VWM" (Liesefeld et al., 2014, p. 327) wherein more successful filtering is indicated by less unnecessary storage. With respect to the bouncer analogy, Liesefeld et al. liken distractor detection to the bouncer's eyes and filter initiation to the bouncer's hands; in their model, the bouncer must first identify the unwanted guests before preventing them from entering the club.

Although these individual pairwise associations, as well as the observed temporal order of the electrophysiological components, are consistent with their proposed model, the authors did not test the path model implied by their causal chain, nor did they evaluate these processes as simultaneous predictors of VWM capacity. Consequently, it remains unclear whether the impact of distractor detection on unnecessary storage is fully explained by prefrontal bias strength, as suggested by the causal chain model. Further, although the authors proposed that "both the bouncer's eyes and hands are vital" (p. 332) to determining individual differences in VWM capacity, it is unclear whether any or all three of the processes comprising efficient filtering uniquely (i.e., when examined as simultaneous predictors) predict VWM capacity.

\section{Reanalysis}

To address this issue, we used the correlations provided in Liesefeld et al. (2014) to test the proposed causal chain among the three filtering-related processes (i.e., distractor detection, filtering initiation, and unnecessary storage) and an additional link from unnecessary storage to VWM capacity (consistent with Awh \& Vogel, 2008; McNab \& Klingberg, 2008). Results are shown in Fig. 2A. This model provided poor fit $\left(\right.$ model $\chi^{2}=8.83, d f=3, p=.03, \mathrm{CFI}=.78, \mathrm{RMSEA}=.22, p$ for close fit $=.05$ ) owing to a large residual association between prefrontal bias signal strength and VWM capacity not accounted for by the model as specified. Thus, a modified model was tested in which an additional direct path from prefrontal bias to VWM capacity was included. This modified model provided excellent model fit (model $\chi^{2}=0.28, d f=2$, $p=.687$, CFI $>.99$, RMSEA $<.01, p$ for close fit $=.88)$. Further, the remaining residual associations (between distractor detection latency and unnecessary storage, and between distractor detection and VWM capacity) were small and non-significant. As shown in Fig. 2B, prefrontal bias strength was a unique predictor of VWM capacity $(p=.002)$, whereas the predictive effect of unnecessary storage was nonsignificant $(p=.49)$. Note that results were consistent when the two remaining predictive paths (from distraction detection to unnecessary storage and to VWM capacity) were added to the model $(d f=0)$; see Fig. $2 \mathrm{C}$ for these results. Critically, the model identifying filtering activity as a direct predictor of VWM capacity explained three times as much variance in individual differences of VWM capacity $\left(R^{2}=.27\right)$ as did the original model attributing that variance to unnecessary storage $\left(R^{2}=.09\right)$.

Consistent with Liesefeld et al.'s proposed causal chain comprising three filtering-related processes, the path model results indicate that distractor detection directly predicted prefrontal bias signal strength, prefrontal signal strength directly predicted unnecessary storage, and this latter path fully accounted for the link between distraction detection and unnecessary storage. With respect to predicting VWM capacity, however, results also indicate that unnecessary storage did not account for the association between the initiation of filtering activity (as reflected by prefrontal bias strength) and VWM capacity. Further, unnecessary storage activity and distractor detection were not unique predictors of VWM capacity independent of filtering initiation. The revised findings thus suggest that of the three filtering-related processes, only prefrontal bias strength was a unique predictor of VWM capacity - not the processes occurring prior to (distraction detection) or subsequent to (unnecessary storage) it in the causal chain.

That is, how effectively targets and distractors are selected is particularly important to VWM capacity, rather than how quickly the distractors are identified. In this regard the present findings suggest a modification to the proposal from Liesefeld et al. (2014) that both the bouncer's eyes and hands are vital to determining individual differences in VWM capacity. Although distractor detection is important for the filtering process (as revealed by the significant link between distraction detection and filtering initiation), distractor detection is not directly or uniquely relevant to determining individual differences in VWM capacity (as revealed by the non-significant link between distractor detection latency and VWM capacity, independent of filtering activity). 
A

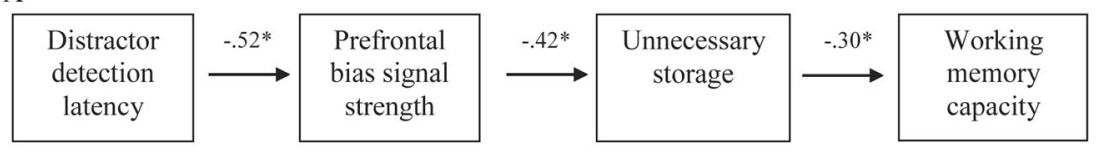

B
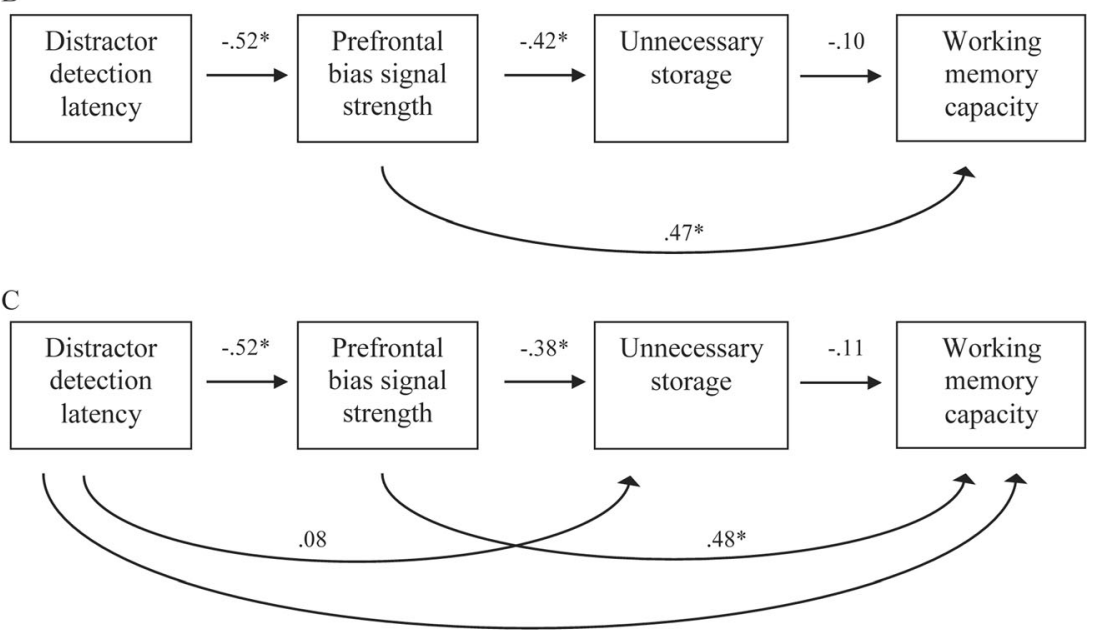

.03

Fig. 2 Results from the re-analysis of the findings presented by Liesefeld et al. (2014). Panel A shows results from the first path model tested. Panel $\mathrm{B}$ shows results from the modified model. Panel $\mathrm{C}$ shows results from a model incorporating all predictive effects. Standardized path coefficients are shown. ${ }^{*} \mathrm{p}<.05$

\section{General discussion: Does the bouncer need a raise?}

Together, the re-analyses from both studies converge on the conclusion that the amount of unwanted information in VWM (as reflected by unnecessary storage) is not the primary determinant of VWM capacity. Rather, when tested alongside neural indicators of filtering preparedness or engagement (occurring temporally prior to the onset of the unnecessary storage activity), it is this activity that is uniquely predictive of VWM capacity. Further, the revised models in which activity associated with filtering directly predicts VWM capacity explained roughly two to three times as much variance in individual differences in VWM capacity as did the original models in which the link between filtering activity and VWM capacity was assumed to be indirect, via unnecessary storage. Accordingly, the present findings help to elucidate the mechanisms underlying individual differences in VWM capacity by ruling out particular stages and types of processing (i.e., unnecessary storage and distraction detection) as unique and direct predictors of VWM capacity, while also identifying filtering-related activity (i.e., filtering preparedness and initiation) as the primary predictor of VWM capacity in these studies.

How could filtering-related activity impact VWM capacity if not through unnecessary storage? The conceptual framework for how unnecessary storage relates to VWM capacity is somewhat murky. Unnecessary storage activity in both
fMRI (McNab \& Klingberg, 2008) and ERPs (Vogel et al., 2005 ) is identified by comparing activity when low numbers of both targets and distractors are present to when only low numbers of distractors are present. The additional "storage"related activity in the presence of distractors indicates a poor selection mechanism, and it is this additional activity that is predictive of VWM capacity. Thus, the conceptual model of unnecessary storage impacting VWM capacity was conceived largely within a framework in which VWM contains a limited number of storage slots; according to this framework, processing of irrelevant (i.e., distractor) information is critical to predicting VWM performance insomuch as unwanted information occupies these limited storage units and restricts the amount of target information that can be stored. However, although it is clear why storing irrelevant distractors should reduce the number of available slots for storing targets, it is unclear how this mechanism applies when only target information is present. That is, if capacity is measured by how many targets can be stored when no distractors are present, how does unnecessary storage impact capacity when no distractors are present? It would appear, therefore, that identifying a mechanism through which target (in addition to distractor) information is processed may have more explanatory power, even within the context of a limited number of storage slots.

Moreover, there is recent debate in the field as to whether, in fact, VWM capacity is limited by the number of storage 
slots alone, thus potentially calling into question the framework by which unnecessary storage limits VWM performance in a capacity limited system. Namely, resource models conceptualize working memory as a limited pool of resources that must be allocated across all (task-relevant) items - as the proportion of resources allocated to each item decreases, the precision or fidelity of those representations decrease (Bays, Catalao, \& Husain, 2009; Bays \& Husain, 2008; Huang, 2010; van den Berg, Shin, Chou, George, \& Ma, 2012). Importantly, the precision of the maintained representations can vary between items (Fougnie, Suchow, \& Alvarez, 2012), trials (van den Berg et al., 2012), by the allocation of attention (Gorgoraptis, Catalao, Bays, \& Husain, 2011), and may be under cognitive control (Machizawa, Goh, \& Driver, 2012), such that precision and the number of items maintained can be traded off against each other (Roggeman, Klingberg, Feenstra, Compte, \& Almeida, 2014). Consequently, although the processing of irrelevant information would reduce the amount of resources allocated to task-relevant information, preparatory activity that is associated with filtering may also be related to VWM performance through the efficient processing of relevant (target) information (e.g., by selectively enhancing the precision of target items through the allocation of resources). For example, one study identified activity in the inferior frontal sulcus that correlates with a trade-off between the number and fidelity of the stored target items (Roggeman et al., 2014). Interestingly, changes in the precision of representations maintained in VWM may occur in the sensory cortex rather than in the storage-related activity associated with the intraparietal sulcus (IPS) (Emrich, Riggall, Larocque, \& Postle, 2013), thereby potentially calling into question the role of delay-period parietal activity in VWM maintenance and representation. Results from the re-analyses presented in the present work do not speak to the slots-versusresources debate, and it is possible that changes in VWM precision via top-down attentional control signals can be accomodated within a slots + resource model (van den Berg et al., 2012; Zhang \& Luck, 2008). Nevertheless, the present results do suggest that understanding precisely how target information is encoded into VWM may be more critical to determining individual differences in VWM than is the unnecessary storage of irrelevant information.

The proposal that filtering preparedness or initiation activity may be related to the processing of targets as well as distractors is also consistent with the hypothesized role of filtering activity as indicative of top-down control over the flow of information into VWM (Awh \& Vogel, 2008; Gazzaley \& Nobre, 2012; McNab \& Klingberg, 2008). From this perspective, filtering activity could reflect general top-down (executive) attentional control mechanisms that are also involved in the selection and encoding of information in VWM. Individual differences in such top-down attentional processes may be responsible for the coordination of filtering and unnecessary storage, as reflected in the association between these two processes, and for their links with VWM capacity. Such an account is consistent with findings indicating that VWM capacity is correlated with the ability to recover from the attentional capture by task-irrelevant distractors (Fukuda \& Vogel, 2009). Thus, the top-down attentional processes that drive efficient filtering may be the same processes that drive the efficient selection and encoding of information, and it is this more general top-down attentional mechanism that underpins individual differences in VWM capacity. In this sense, "filtering set activity" and the "prefrontal bias signal" may not reflect activity specific to filtering, but rather activity related to a broader top-down mechanism that is involved with the processing of both relevant and irrelevant information.

Interestingly, recent evidence may provide some insight into the precise mechanisms that may mediate the top-down processing of targets in VWM. Feredoes and colleagues (Feredoes, Heinen, Weiskopf, Ruff, \& Driver, 2011) examined the role of the dorsolateral prefrontal cortex (DLPFC) in processing targets and distractors. Participants were instructed to maintain images of faces or houses, and on some of the trials images from the other category could be presented as distractors during the delay. Critically, when the DLPFC was stimulated with transcranial magnetic stimulation (TMS) during the delay period, an increase in activity was observed in the areas of visual cortex involved in processing the target stimuli (e.g., the fusiform face area). Thus, the DLPFC may serve as the source of a top-down signal that is involved in the enhancement of target stimuli.

In many ways, our observation that filtering-related activity predicts VWM capacity independent of unnecessary storage activity is subject to the same limitations as the model it disconfirms. Whereas the original model stated that the unnecessary storage of task-irrelevant distractors predicts VWM capacity - even when capacity is measured in the absence of distractors - the revised model suggests that filtering-related activity predicts VWM capacity even in the absence of filtering. That is, the filtering-related activity in the two studies re-examined in the present work was operationalized as activity present during active filtering and absent when filtering is not required. Thus, it is unclear how this activity (and the process(es) reflected by it) might affect VWM capacity when filtering is not required. Consequently, it will be critical for future studies to determine how to identify filtering-related activity in the absence of distractors, or how to better operationalize the activity observed during filtering. A potential candidate for such activity may be provided by a study that found a positive correlation between VWM capacity (which was measured both by behavioral performance and by the amount of BOLD signal observed in the IPS) and activity in the DLPFC (Edin et al., 2009). The authors of this study suggested that VWM capacity was partly determined by topdown control driven by activity originating in the DLPFC, 
even when distractors were not present. In other words, consistent with the analysis reported here, findings from Edin et al. suggest that individual differences in VWM performance may be explained by top-down signals driving target processing, rather than by unnecessary storage. Importantly, the topdown activity reported by Edin et al. was modulated by working memory load, suggesting that top-down signals from DLPFC are tied to variations in the amount of attentional control required. Thus, one possible reason why tasks that require filtering elicit a strong top-down control signal is because filtering requires greater attentional control. However, further research is required to fully establish the links between DLPFC activity, VWM capacity, and filtering.

It is important to stress that although the models tested here provide strong evidence against the unique role of unnecessary storage in predicting VWM capacity, we acknowledge that the revised model, too, may be incomplete or incorrect, as may be revealed in future studies based on additional experimental evidence. For example, future studies may identify more sensitive or valid measures of the key underlying cognitive processes under consideration in the present work; in such cases, further examination of the models examined here would be valuable. In addition, there are limitations to the path analytic approach we employed. For example, we do not know the reliabilities of the various measures, nor have we tested possible non-linear effects, both of which could affect the outcome of these analyses. Furthermore, the modest sample sizes of both studies we re-examined likely attenuated the statistical power to detect as statistically significant relatively small predictive effects. ${ }^{1}$ Moreover, it is possible that activity that has previously been identified as reflecting "unnecessary storage" or "filtering initiation" reflect as-of-yet undefined processes or cognitive mechanisms. Finally, further studies are required to determine more precisely the relationship between top-down signals (e.g., DLPFC activity), the processing of targets and distractors, and unnecessary storage; indeed, the results presented here highlight the importance of fully testing the different possible models moving forward, ideally through directly evaluating competing models.

\footnotetext{
$\overline{1}$ To evaluate the robustness of our findings, we re-estimated the parameters in each model (path coefficients, $p$ values) using a bootstrapping approach and based on bias-corrected estimates from 1,000 resamples. Results were consistent with those presented in the main text. These bootstrapping results indicate that our findings are not overly biased by the relatively small sample sizes of the studies we re-analyzed. However, the statistical power to detect relatively smaller predictive effects was limited by the sample sizes; for example, a sample size of approximately 100 participants would be needed to find the path from unnecessary storage to working memory capacity shown in Fig. 1B to be statistically significant.
}

\section{Conclusion}

Findings from our re-analyses indicate that Awh and Vogel (2008) were correct in emphasizing the importance of a good bouncer (i.e., a strong filtering mechanism) in determining the success of the VWM nightclub. And yet the value of the activity associated with the initial stages in filtering is not limited to or explained by the relationship between unnecessary storage and VWM capacity. Rather, activity associated with filtering preparedness or initiation appears to play a more central role in predicting individual differences in VWM capacity than has been previously recognized. The precise nature of this activity (e.g., as a determinant of efficient selection and encoding of relevant information, or as an indicator of a more general top-down attentional control process) has yet to be clarified. To this end, our work provides novel conceptual and empirical insights concerning the roles of filtering-related activity and unnecessary storage in understanding individual differences in VWM capacity.

Acknowledgments The authors would like to thank Naseem-AlAidroos, Karen Arnell, Jeffrey S. Johnson, Steve Luck, and two anonymous reviewers for helpful feedback. This work was supported by a Natural Sciences and Engineering Research Council of Canada grant [435945] awarded to S.M.E.

\section{References}

Awh, E., \& Vogel, E. K. (2008). The bouncer in the brain. Nature Neuroscience, 11(1), 5-6. doi:10.1038/nn0108-5

Bays, P. M., \& Husain, M. (2008). Dynamic shifts of limited working memory resources in human vision. Science, 321(5890), 851-854. doi:10.1126/science. 1158023

Bays, P. M., Catalao, R. F. G., \& Husain, M. (2009). The precision of visual working memory is set by allocation of a shared resource. Journal of Vision, 9(10), 7.1-11. doi:10.1167/9.10.7

Cowan, N. (2001). The magical number 4 in short-term memory: a reconsideration of mental storage capacity. Behavioral and Brain Sciences, 24(1), 87-114. discussion 114-85.

Edin, F., Klingberg, T., Johansson, P., McNab, F., Tegnér, J., \& Compte, A. (2009). Mechanism for top-down control of working memory capacity. Proceedings of the National Academy of Sciences of the United States of America, 106(16), 6802-6807. doi:10.1073/pnas. 0901894106

Emrich, S. M., Riggall, A. C., Larocque, J. J., \& Postle, B. R. (2013). Distributed patterns of activity in sensory cortex reflect the precision of multiple items maintained in visual short-term memory. The Journal of Neuroscience, 33(15), 6516-6523. doi:10.1523/ JNEUROSCI. 5732-12.2013

Feredoes, E., Heinen, K., Weiskopf, N., Ruff, C., \& Driver, J. (2011). Causal evidence for frontal involvement in memory target maintenance by posterior brain areas during distracter interference of visual working memory. Proceedings of the National Academy of Sciences of the United States of America, 108(42), 17510-17515. doi:10. 1073/pnas.1106439108 
Fougnie, D., Suchow, J. W., \& Alvarez, G. A. (2012). Variability in the quality of visual working memory. Nature Communications, 3, 1229. doi:10.1038/ncomms 2237

Fukuda, K., \& Vogel, E. K. (2009). Human variation in overriding attentional capture. The Journal of Neuroscience, 29(27), 8726-8733. doi:10.1523/JNEUROSCI. 2145-09.2009

Gazzaley, A., \& Nobre, A. C. (2012). Top-down modulation: bridging selective attention and working memory. Trends in Cognitive Sciences, 16(2), 129-135. doi:10.1016/j.tics.2011.11.014

Gorgoraptis, N., Catalao, R. F. G., Bays, P. M., \& Husain, M. (2011). Dynamic updating of working memory resources for visual objects. The Journal of Neuroscience, 31(23), 8502-8511. doi:10.1523/ JNEUROSCI. 0208-11.2011

Huang, L. (2010). Visual working memory is better characterized as a distributed resource rather than discrete slots. Journal of Vision, 10(14), 8. doi:10.1167/10.14.8

Liesefeld, A. M., Liesefeld, H. R., \& Zimmer, H. D. (2014). Intercommunication between prefrontal and posterior brain regions for protecting visual working memory from distractor interference. Psychological Science, 25(2), 325-333. doi:10.1177/ 0956797613501170

Luck, S. J., \& Vogel, E. K. (2013). Visual working memory capacity: from psychophysics and neurobiology to individual differences. Trends in Cognitive Sciences, 17(8), 391-400. doi:10.1016/j.tics. 2013.06.006

Machizawa, M. G., Goh, C. C. W., \& Driver, J. (2012). Human visual short-term memory precision can be varied at will when the number of retained items is low. Psychological Science, 23(6), 554-559. doi: 10.1177/0956797611431988

McCollough, A. W., Machizawa, M. G., \& Vogel, E. K. (2007). Electrophysiological measures of maintaining representations in visual working memory. Cortex; a Journal Devoted to the Study of the Nervous System and Behavior, 43(1), 77-94.

McNab, F., \& Klingberg, T. (2008). Prefrontal cortex and basal ganglia control access to working memory. Nature Neuroscience, 11(1), 103-108.

Roggeman, C., Klingberg, T., Feenstra, H. E. M., Compte, A., \& Almeida, R. (2014). Trade-off between capacity and precision in visuospatial working memory. Journal of Cognitive Neuroscience, 26(2), 211-222. doi:10.1162/jocn_a_00485

Van den Berg, R., Shin, H., Chou, W.-C., George, R., \& Ma, W. J. (2012). Variability in encoding precision accounts for visual short-term memory limitations. Proceedings of the National Academy of Sciences of the United States of America, 109(22), 8780-8785. doi:10.1073/pnas.1117465109

Vogel, E. K., Mccollough, A. W., \& Machizawa, M. G. (2005). Neural measures reveal individual differences in controlling access to working memory. Nature, 438(7067), 500-503. doi:10.1038/ nature 04171

Zhang, W., \& Luck, S. J. (2008). Discrete fixed-resolution representations in visual working memory. Nature, 453(7192), 233-235. doi:10. 1038/nature06860 